\title{
Optical clearing for luminal organ imaging with ultrahigh-resolution optical coherence tomography
}

\author{
Yanmei Liang \\ Wu Yuan \\ Jessica Mavadia-Shukla \\ Xingde Li
}




\title{
Optical clearing for luminal organ imaging with ultrahigh-resolution optical coherence tomography
}

\author{
Yanmei Liang, ${ }^{a, b, *, \dagger}$ Wu Yuan, ${ }^{a, \dagger}$ Jessica Mavadia-Shukla, ${ }^{a}$ and Xingde $\mathrm{Li}^{\mathrm{a}}$ \\ a Johns Hopkins University, Department of Biomedical Engineering, Baltimore, Maryland 21205, United States \\ bNankai University, Institute of Modern Optics, Key Laboratory of Optical Information Science and Technology, Ministry of Education, \\ Tianjin 300071, China
}

\begin{abstract}
The imaging depth of optical coherence tomography (OCT) in highly scattering biological tissues (such as luminal organs) is limited, particularly for OCT operating at shorter wavelength regions (such as around $800 \mathrm{~nm}$ ). For the first time, the optical clearing effect of the mixture of liquid paraffin and glycerol on luminal organs was explored with ultrahigh-resolution spectral domain OCT at $800 \mathrm{~nm}$. Ex vivo studies were performed on pig esophagus and bronchus, and guinea pig esophagus with different volume ratios of the mixture. We found that the mixture of $40 \%$ liquid paraffin had the best optical clearing effect on esophageal tissues with a short effective time of $\sim 10 \mathrm{~min}$, which means the clearing effect occurs about 10 min after the application of the clearing agent. In contrast, no obvious optical clearing effect was identified on bronchus tissues. ๑ 2016 Society of PhotoOptical Instrumentation Engineers (SPIE) [DOI: 10.1117/1.JBO.21.8.081211]
\end{abstract}

Keywords: optical coherence tomography; optical clearing technique; luminal organs.

Paper 160061SSRR received Jan. 30, 2016; accepted for publication Jun. 3, 2016; published online Jun. 23, 2016.

\section{Introduction}

Optical coherence tomography (OCT) affords high-resolution imaging of tissue microanatomy in vivo. However, the limited imaging depth due to the high scattering of light in biological tissues hinders its potential applications, where a deeper imaging depth is desired. To achieve a better imaging depth, optical clearing is regarded as the most effective method. It is generally considered that optical clearing results from collective effects including the refractive index matching effect, the cell dehydration effect, and the increased collagen solubility due to the osmotic property of optical clearing agents (OCAs), which leads to a deeper penetration of light in biological tissues. ${ }^{1-9}$ Recently, increasing research interest has been witnessed on the efforts to develop and validate new and more effective OCAs.

New OCAs, mixtures of two or more agents, capable of extending imaging depth or even making tissue transparent, such as SeeDB, ${ }^{10}$ benzyl alcohol and benzyl benzoate, ${ }^{11}$ Scale, ${ }^{12}$ and the mixture of tetrahydrofuran and dibenzyl ether, ${ }^{13}$ were developed and successfully demonstrated in several ex vivo mouse brain tissue studies. With these new OCAs, a few days' process for dehydration and maceration of biological tissues was needed for optical clearing to take effect. In contrast, a new method for three-dimensional imaging of solvent-cleared organs successfully reduced the process time to several hours. ${ }^{14}$ However, all these new OCAs are toxic and suitable only for ex vivo applications.

For in vivo applications, an ideal OCA should be safe to use and has a short effective time (which is defined as the time for the optical clearing effect to take place after the application of the agent). To this end, some biocompatible hyperosmotic and hydrophilic chemical agents, e.g., polyethylene glycol, ${ }^{15}$ glucose, ${ }^{16,17}$ glycerol, ${ }^{18,19}$ and propylene glycol, ${ }^{20}$ have been tested.

*Address all correspondence to: Yanmei Liang, E-mail: ymliang@ nankai.edu .cn

†These authors contributed equally to this work.
The hyperosmotic property leads to an efficient diffusion of these biocompatible OCAs into biological tissues. For example, an effective time of about $50 \mathrm{~min}$ was reported in an in vivo study on human skin by using glycerol, which is one of the most commonly used biocompatible agents for optical clearing. ${ }^{1}$ To further shorten the effective time, some physical methods, such as sonophoresis with ultrasound, ${ }^{21}$ rubbing with sandpaper, ${ }^{22}$ or puncturing with microneedle roller, ${ }^{23}$ were developed to aid the diffusion of glycerol through the dense stratum corneum (SC) of skin. However, these methods cannot be directly or conveniently applied to luminal organs in vivo. Alternatively, it was proposed to optimize the penetration efficiency of glycerol in biological tissues by incorporating a permeation enhancer, such as oleic acid, ${ }^{24}$ dimethyl sulfoxide, ${ }^{24-26}$ azone, ${ }^{27}$ thiazone, ${ }^{28,29}$ or liquid paraffin. ${ }^{30-33}$ The enhanced optical clearing effect of liquid paraffin and glycerol mixture was validated on human skin, where a much shorter effective time of $\sim 10$ min was achieved. ${ }^{30-32}$ Furthermore, as a lipophilic agent, it was found that liquid paraffin can greatly relieve the severe dehydration effect caused by glycerol, ${ }^{30,31}$ which induces tissue warping and might not be safe to use for certain clinical applications. It is noteworthy that liquid paraffin has been used in cosmetics and food applications for $>100$ years. ${ }^{34}$ Liquid paraffin has also been approved by the Food and Drug Administration as a safe pharmaceutical vehicle and is listed in many different pharmacopeia. ${ }^{34}$

In this paper, we studied the optical clearing effect of liquid paraffin and glycerol mixture on luminal organs ex vivo with a spectral domain optical coherence tomography (SD-OCT) system operating at $800 \mathrm{~nm}$. Our experimental results verified that the mixture of $40 \%$ liquid paraffin had the optimum optical clearing effect on both guinea pig and pig esophagus tissues with an effective time as short as only about $10 \mathrm{~min}$, whereas no obvious optical clearing effect was found on pig bronchus tissues. 


\section{Materials and Methods}

\subsection{Materials}

Esophagus and bronchus tissues from four pigs and esophagus tissues from one guinea pig were harvested for this study right after the animals were euthanized for other purposes approved by the Animal Care and Use Committee at the Johns Hopkins University. The luminal tissues were cut into small pieces of $\sim 1 \times 1 \mathrm{~cm}$ immediately after tissue harvest. Fresh specimens were submerged in phosphate-buffered saline (PBS) solution and kept in a $4^{\circ} \mathrm{C}$ refrigerator for no more than $5 \mathrm{~h}$ before use. After being well mixed by stirring for at least $10 \mathrm{~min}$, about $40 \mu \mathrm{l}$ of the liquid paraffin and glycerol mixture with a volume ratio of $20 \%$ to $60 \%$ were immediately applied on the inner surface of the luminal tissues using a syringe needle. It was found that a well-mixed liquid paraffin/glycerol mixture took over $4 \mathrm{~h}$ to have a complete phase separation, while in our experiments each test lasted for at most $50 \mathrm{~min}$ so as to greatly mitigate the influence of phase separation. Herein, the volume ratio was calculated as $V_{\mathrm{lp}} /\left(V_{\mathrm{lp}}+V_{\mathrm{gl}}\right)$, where $V_{\mathrm{lp}}$ is the volume of liquid paraffin and $V_{\mathrm{gl}}$ is the volume of glycerol.

\subsection{Imaging System}

As shown in Fig. 1, our SD-OCT system adopted a customized broadband linear-in-wavenumber spectrometer to afford an imaging depth of $\sim 1.2 \mathrm{~mm}$, the detail of which was reported previously. ${ }^{35,36}$ A homemade Ti:Sapphire laser with a central wavelength of $\sim 825 \mathrm{~nm}$ and a 3-dB bandwidth of $\sim 150 \mathrm{~nm}$ was used to achieve an axial resolution of $\sim 2.8 \mu \mathrm{m}$ (in air). Two galvonometer scanning mirrors were employed in the sample arm for performing $X-Y$ two-dimensional beam scanning. A scan lens was used to afford a lateral resolution of $\sim 16 \mu \mathrm{m}$. Sample imaging was performed at $70 \mathrm{k} \mathrm{A-scans/s.}$

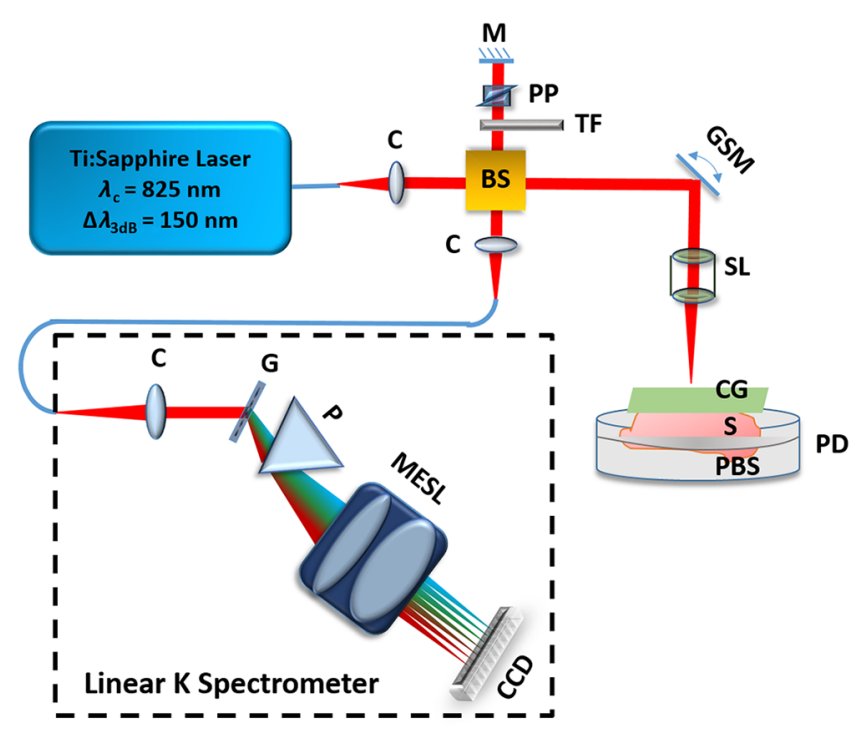

Fig. 1 Schematic of the ultrahigh-resolution spectral-domain OCT imaging system. BS, beam splitter; $\mathrm{C}$, multielement achromatic collimator; charge coupled device (CCD), line scan CCD; CG, cover glass; G, grating; GSM, galvonometer scanning mirrors; M, mirror; MESL, multielement scan lens; $\mathrm{P}$, linear $\mathrm{K}$ mapping prism; $\mathrm{PP}$, prism pair; S, specimen; PBS, phosphate-buffered saline; PD, Petri dish; SL, scan lens; and TF, tunable filter.

\subsection{Experiment Design}

All imaging experiments were performed at room temperature. As shown in Fig. 1, the lower part of the specimen was immersed in a small volume of PBS solution during imaging to prevent the specimen from dehydration. The liquid paraffin/ glycerol mixture was applied evenly on the tissue surface and sealed between a cover glass and the specimen surface. In our control experiments, PBS instead of the liquid paraffin/glycerol mixture was applied on the tissue surface. The diffusion process of the mixture was studied by B-scan (cross sectional) imaging of the tissue at a given place every 5 min for a total of 50-min duration to understand the efficacy of the optical clearing effect. Each B-scan image consisted of $2048 \times 2048$ pixels (lateral $\times$ axial) corresponding to a physical image size of $2 \mathrm{~mm} \times 1.2 \mathrm{~mm}$. Each dataset consisted of $20 \mathrm{~B}$-scan images, which were used for averaging to reduce speckle noise.

\subsection{Merit Metrics}

B-scan images provided direct and qualitative visual assessment of the optical clearing effect. To quantify such effect, some metrics have been proposed previously. ${ }^{17,30,31,37-40}$ Considering the heterogeneous nature of tissue structures, tissue optical property, and their potential changes during the process of OCA application and diffusion, evaluating the optical property change at one fixed tissue depth alone would not be sufficiently accurate. Therefore, in addition to optical property, we proposed a merit metric called intensity ratio of regions (RIR), ${ }^{30,31}$ which calculates the ratio of average intensities of a designated region inside the tissue and a region nearby the tissue surface, and it is defined as ${ }^{30,31}$

$$
\mathrm{RIR}=\frac{\frac{1}{N_{1}} \sum_{x, y \in \text { internal }} I(x, y)}{\frac{1}{N_{2}} \sum_{x, y \in \text { surface }} I(x, y)},
$$

where $N_{1}$ and $N_{2}$ are the number of pixels of the two regions of interest, respectively, and $I(x, y)$ represents the OCT intensity at position $(x, y)$. The time-dependent optical clearing effect was assessed with RIR and benchmarked with the RIR value a minute after the application of the clearing agent to obtain the relative RIRs. Generally, a larger relative RIR value means a better optical clearing effect.

\section{Experimental Results}

As shown in Fig. 2, a series of representative B-scan images at a fixed location on the guinea pig esophagus were displayed over a time window of about $50 \mathrm{~min}$ after the application of the liquid paraffin (at 30\% volume concentration) and glycerol mixture. Since it was difficult to ensure the same B-scan position before and after the application of the clearing agent, the B-scan image used as a benchmark was acquired at the first minute after the application of the mixture. As shown in Fig. 2(b), the optical clearing effect of the mixture on the esophagus tissue was evident. The increased transparency of upper layers, such as epithelium and lamina propria, led to a pronounced muscle layer at only $10 \mathrm{~min}$ after the application of the paraffin-glycerol mixture.

To quantitatively study the optical clearing effect and find the optimum paraffin-glycerol volume ratio, mixtures of $30 \%, 40 \%$, and $50 \%$ of liquid paraffin were used on pig esophagus tissues. 


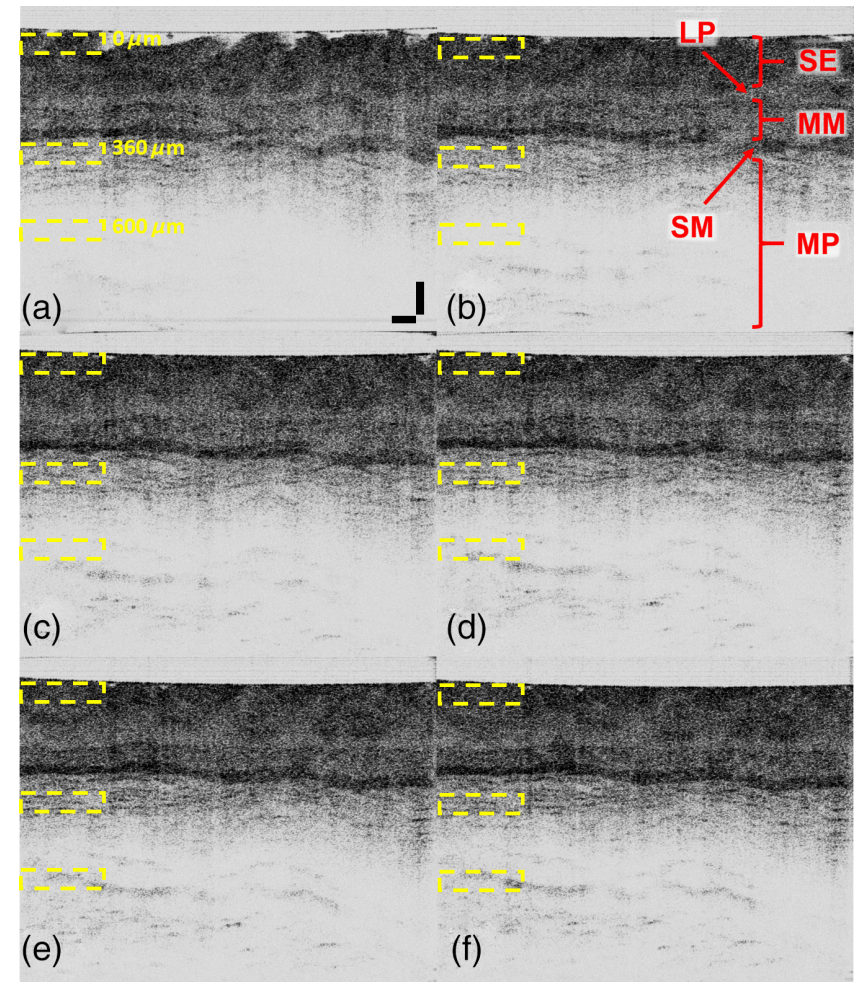

Fig. 2 Representative B-scan images of guinea pig esophagus at (a) $1 \mathrm{~min}$, (b) $10 \mathrm{~min}$, (c) $20 \mathrm{~min}$, (d) $30 \mathrm{~min}$, (e) $40 \mathrm{~min}$, and (f) $50 \mathrm{~min}$, after the application of $30 \%$ paraffin/glycerol mixture. Yellow rectangle boxed regions in (a) correspond to $0, \sim 360$, and $\sim 600 \mu \mathrm{m}$ below the tissue surface, respectively, and are selected to calculate RIRs. SE, stratified epithelium; LP, lamina propria; MM, muscularis mucosae; SM, submucosa; and MP, muscularis propria. Scale bars in (a): $100 \mu \mathrm{m}$.

Small image regions of $400 \times 100$ pixels (lateral $\times$ axial) near the tissue surface and at different depths were used to calculate the RIRs. In this study, regions starting from pixel 1, 600, and 1000 below the tissue surface along the imaging depth were selected, which correspond to $0, \sim 360$, and $\sim 600 \mu \mathrm{m}$ below the tissue surface, respectively, as shown in Fig. 2.

As shown in Fig. 3, the relative RIR value [calculated by using Eq. (1) and then dividing by the RIR benchmark value] was studied at different tissue depths and plotted along with time after the application of the mixture. As mentioned earlier, the RIR benchmark value was the one calculated over the same image region but at the first minute after the mixture application. The standard deviations were calculated with three pig esophagus tissues and displayed as error bars in Fig. 3. It is found that optical clearing by the mixture occurred on the esophagus tissue with the relative RIRs $>1$ for all paraffin volume ratios used in this study. The bigger the relative RIR is, the higher the improvement is for the intensity value at the internal position relative to that at the surface, which means a better optical clearing effect. The best optical clearing effect (represented by the highest relative RIR) was achieved with a paraffin volume ratio of $40 \%$ by comparing the relative RIR values at different tissue depths (in particular at depths of $\sim 360 \mu \mathrm{m}$ ), which is consistent with the observation reported in Ref. 30. As seen from Fig. 3, the optical clearing effect took place very rapidly during the first $10 \mathrm{~min}$ for all tested mixtures on the esophagus tissue. This is a remarkable property, which may be very useful for the in vivo applications, where a fast optical clearing effect is
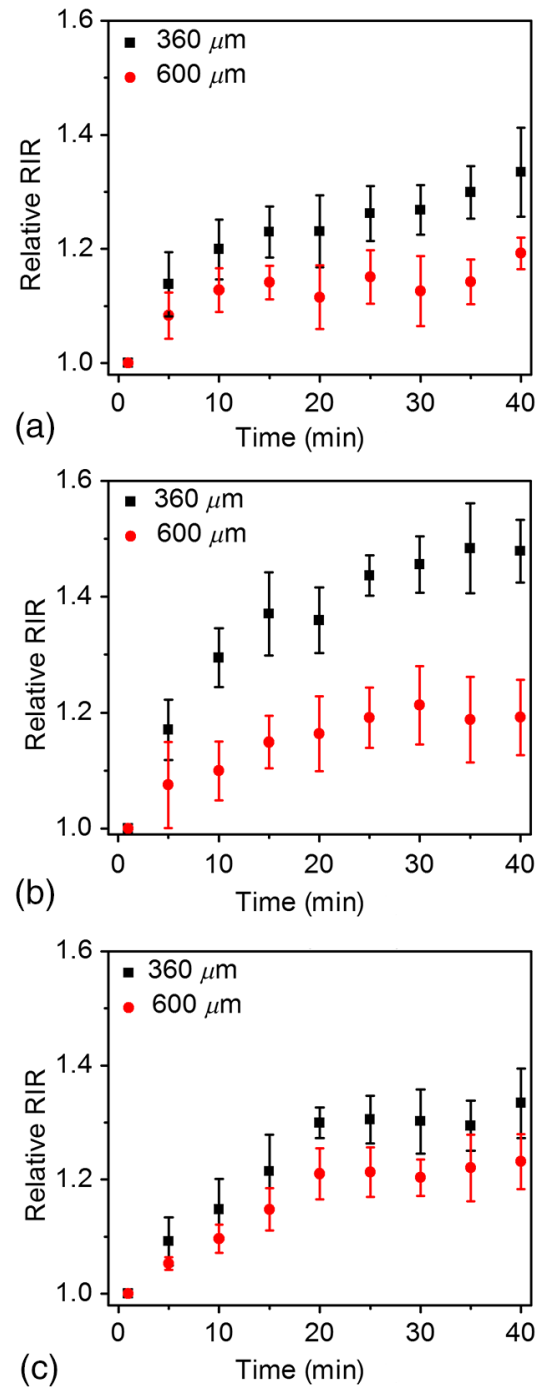

Fig. 3 Relative RIR variation versus time after applying the liquid paraffin and glycerol mixture on pig esophagus tissues with different volume ratios of (a) $30 \%$, (b) $40 \%$, and (c) $50 \%$. Red solid circles and black solid squares correspond to different depths in the tissue.

needed. Validation experiments using guinea pig esophagus tissues revealed the similar results (results not shown).

To further scrutinize the consequences of optical clearing, depth-resolved optical properties (i.e., the attenuation coefficient) of the esophagus tissue have been studied versus time after the application of the liquid paraffin/glycerol mixture. The algorithm used for quantifying the optical attenuation coefficient is similar to the one reported in Ref. 41. In essence, the depthresolved attenuation coefficients for each A-scan were calculated with the following equation:

$\mu[i] \approx \frac{I[i]}{\Delta \cdot \sum_{i+1}^{\infty} I[i]}$,

where $\mu[i]$ is the attenuation coefficient at the $i$ 'th pixel of a given A-scan, which is defined as the averaged attenuation over the pixel size of $\Delta . I[i]$ is the OCT intensity at the $i$ 'th pixel of that A-scan. It is noteworthy that Eq. (2) is slightly different from the one reported in Ref. 41 by assuming the OCT 


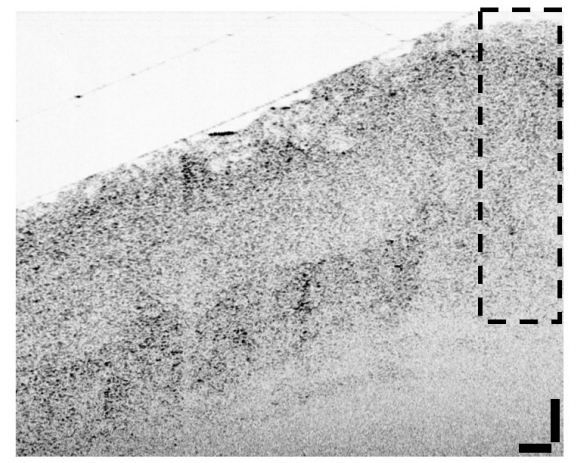

(a)

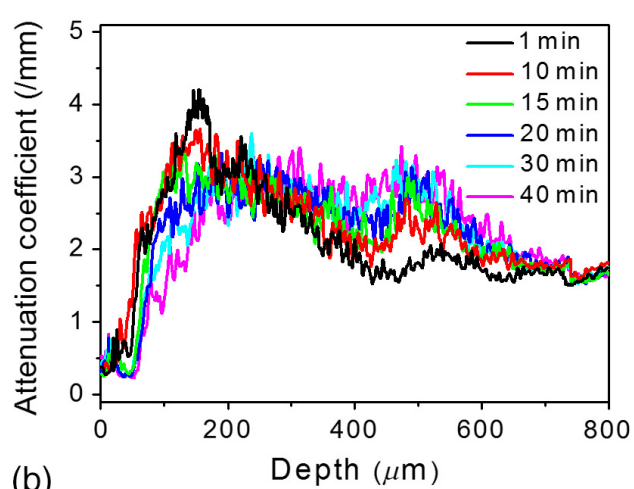

(b)

Fig. 4 (a) Representative attenuation image of the pig esophagus tissue $10 \mathrm{~min}$ after the application of $40 \%$ paraffin/glycerol mixture. (b) The time-dependent change of the averaged attenuation coefficient along depth within a region of interest [boxed by a black rectangle with $400 \times 1300$ pixels in (a)]. Curves of different colors correspond to different times after the application of the mixture. Scale bars in (a): $100 \mu \mathrm{m}$.

signal of each A-scan follows $I[i] \propto e^{-\mu(z) \cdot z}$, where $\mu(z)$ is the attenuation coefficient at medium depth of $z$.

A representative attenuation image of the pig esophagus tissue at $10 \mathrm{~min}$ after the application of a $40 \%$ paraffin/glycerol mixture is shown in Fig. 4(a). As highlighted with a dashed line rectangle in Fig. 4(a), a region of interest with $400 \times$ 1300 pixels (lateral $\times$ axial) was selected to calculate the averaged attenuation coefficient along the A-scan. A significant time-dependent change on the attenuation coefficient was observed as shown in Fig. 4(b). It was found that the attenuation coefficient decreased gradually at the region near the tissue surface and increased at the deeper tissue regions with time. This change eventually led to a decreased attenuation inhomogeneity of the esophagus tissue with the most dynamic change happening during the first $10 \mathrm{~min}$ after the application of the optical clearing mixture.

To study the optical clearing effect of liquid paraffin and glycerol mixture on other internal organs, we further tested

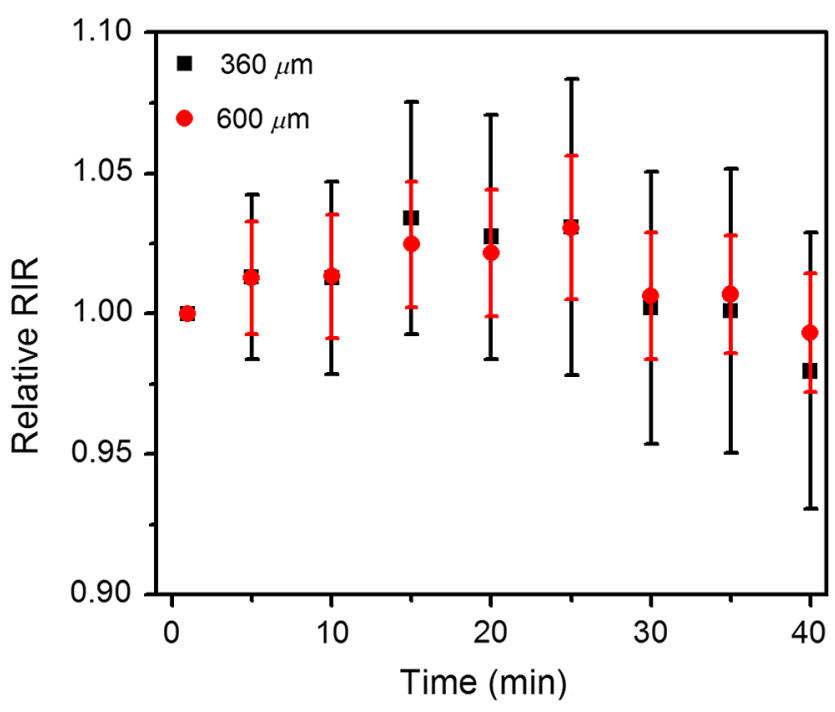

Fig. 5 Relative RIR versus time calculated from the B-scan images of the pig bronchus tissue applied with $30 \%$ paraffin/glycerol mixture. Red solid circles and black solid squares correspond to different depths in the tissue. The optical clearing effect of the agent on the bronchus tissue is not evident. the pig bronchus tissues. No significant optical clearing effect was observed for all tested mixtures with different volume ratios. As shown in Fig. 5 (for a representative 30\% paraffin/ glycerol volume ratio), the relative RIR values changed only slightly with time at different tissue depths. The error bars were calculated with three pig bronchus tissues. It is also noteworthy that in the control experiments no optical clearing effect of PBS was observed for either esophagus or bronchus tissues.

\section{Discussions and Conclusion}

It has been proved that lipophilic agents, such as liquid paraffin, are capable of penetrating epithelium tissue and can serve as a carrier for drug delivery. ${ }^{34,42-44}$ Our experimental results verified that liquid paraffin can facilitate the penetration of glycerol through the dense stratified squamous epithelium of esophagus tissue into deeper layers and work collaboratively with glycerol for tissue index matching. This effect was also confirmed in our previous studies on human skin, where a significantly enhanced optical clearing effect was found by using liquid paraffin to aid the penetration of glycerol through the SC layer of skin. ${ }^{30-32}$ The reason that this facilitation effect does not work for bronchus tissue may be due to the difficulty for the mixture to penetrate the cartilage rich in pig bronchus.

Compared with our previous studies, where a 1500-nm light source was used, higher relative RIR values (i.e., more pronounced optical clearing effect) were found in this study with an 800-nm SD-OCT system. This finding coincides with many previous publications reporting a better optical clearing effect at shorter wavelengths. ${ }^{20,26,28}$ Furthermore, it was found that a fine balance between the dehydration effect of glycerol and the water retention capability of the liquid paraffin helped avoid tissue deformation when using the mixture of $40 \%$ liquid paraffin. This feature is very useful for potential in vivo applications. In contrast, it was also noted that with the mixture of a low-volume ratio, such as $20 \%$, the esophagus tissues showed some obvious warping of tissue surface due to the dominant dehydration effect of glycerol.

The phenomena of a decrease in scattering in upper layers and an increase in scattering in lower layers with time in Fig. 4 prove the attenuation coefficient tends to become homogeneous. From Fig. 4, we can see the attenuation coefficients that have the most dynamic change in the first 10 min after the application of the optical clearing mixture, which means 
glycerol rapidly penetrates into inner tissue with the help of liquid paraffin. After $20 \mathrm{~min}$, they change slowly with time until they become homogeneous. This change can be attributed to the index matching effect of the liquid paraffin/glycerol mixture, which leads to more homogeneous tissue refractive index that in turn reduces tissue attenuation (mainly scattering) and consequently enhances light penetration in the tissue.

In summary, the optical clearing effect of the liquid paraffin/ glycerol mixture on luminal organs, such as the esophagus and bronchus, was studied for the first time at $800-\mathrm{nm}$ wavelength region with an ultrahigh-resolution SD-OCT system. A significant optical clearing effect on esophagus tissues was observed with a short effective time of $\sim 10 \mathrm{~min}$, whereas no obvious optical clearing effect was found on bronchus tissues. We validated that the mixture of $40 \%$ liquid paraffin afforded the optimum optical clearing effect on the esophagus tissues. These findings will facilitate the use of the liquid paraffin/glycerol mixture in the in vivo endoscopic imaging of lumen organs in the future.

\section{Acknowledgments}

This study has been funded by the National Institutes of Health of the United States (under the Grant No. R01HL121788-X.D. L.), the Coulter Foundation (X.D.L.), and the National Natural Science Foundation of China (under the Grant No. 11374167Y.M.L.). The authors thank Xiaoyun Yu and Shaoyong Yu for providing the guinea pig tissue samples.

\section{References}

1. V. V. Tuchin, "Optical clearing of tissues and blood using the immersion method," J. Phys. D Appl. Phys. 38(15), 2497-2518 (2005).

2. X. Wen et al., "In vivo skin optical clearing by glycerol solutions: mechanism," J. Biophotonics 3(1-2), 44-52 (2010).

3. M. Brezinski et al., "Index matching to improve optical coherence tomography imaging through blood," Circulation 103(15), 19992003 (2001).

4. C. G. Rylander et al., "Dehydration mechanism of optical clearing in tissue," J. Biomed. Opt. 11(4), 041117 (2006).

5. D. Zhu et al., "Recent progress in tissue optical clearing," Laser Photonics Rev. 7(5), 732-757 (2013).

6. A. T. Yeh et al., "Reversible dissociation of collagen in tissues," J. Invest. Dermatol. 121(6), 1332-1335 (2003).

7. J. Hirshburg et al., "Collagen solubility correlates with skin optical clearing," J. Biomed. Opt. 11(4), 040501 (2006).

8. J. Gallwas et al., "Effect of optical clearing agents on optical coherence tomography images of cervical epithelium," Lasers Med. Sci. 30(2), 517-525 (2015)

9. R. K. Wang et al., "Investigation of optical clearing of gastric tissue immersed with hyperosmotic agents," IEEE J. Select. Top. Quantum Electron. 9(2), 234-242 (2003).

10. M.-T. Ke, S. Fujimoto, and T. Imai, "SeeDB: a simple and morphologypreserving optical clearing agent for neuronal circuit reconstruction," Nat. Neurosci. 16(8), 1154-1161 (2013).

11. H.-U. Dodt et al., "Ultramicroscopy: three-dimensional visualization of neuronal networks in the whole mouse brain," Nat. Methods 4(4), 331336 (2007).

12. H. Hama et al., "Scale: a chemical approach for fluorescence imaging and reconstruction of transparent mouse brain," Nat. Neurosci. 14(11), 1481-1488 (2011).

13. K. Becker et al., "Chemical clearing and dehydration of GFP expressing mouse brains," PLoS One 7(3), e33916 (2012).

14. A. Ertürk et al., "Three-dimensional imaging of solvent-cleared organs using 3DISCO," Nat. Protoc. 7(11), 1983-1995 (2012).

15. V. V. Tuchin et al., "Light propagation in tissues with controlled optical properties," J. Biomed. Opt. 2(4), 401-417 (1997).
16. A. N. Bashkatov et al., "Optical clearing of skin tissue produced by application of glucose solution: in vivo study," Proc. SPIE 6163, 616313 (2006).

17. E. A. Genina et al., "Optical clearing of the eye sclera in vivo caused by glucose," Quantum Electron. 36(12), 1119-1124 (2006).

18. R. K. Wang et al., "Concurrent enhancement of imaging depth and contrast for optical coherence tomography by hyperosmotic agents," J. Opt. Soc. Am. B 18(7), 948-953 (2001).

19. J. W. Fluhr, R. Darlenski, and C. Surber, "Glycerol and the skin: holistic approach to its origin and functions," Br. J. Dermatol. 159(1), 23-34 (2008).

20. X. Xu and Q. Zhu, "Feasibility of sonophoretic delivery for effective skin optical clearing," IEEE Trans. Biomed. Eng. 55(4), 1432-1437 (2008).

21. H. Zhong et al., "In vitro study of ultrasound and different-concentration glycerol-induced changes in human skin optical attenuation assessed with optical coherence tomography," J. Biomed. Opt. 15(3), 036012 (2010).

22. O. Stumpp, B. Chen, and A. J. Welch, "Using sandpaper for noninvasive transepidermal optical skin clearing agent delivery," J. Biomed. Opt. 11(4), 041118 (2006).

23. J. Yoon et al., "Enhancement of optical skin clearing efficacy using a microneedle roller," J. Biomed. Opt. 13(2), 021103 (2008).

24. J. Jiang and R. K. Wang, "Comparing the synergistic effects of oleic acid and dimethyl sulfoxide as vehicles for optical clearing of skin tissue in vitro," Phys. Med. Biol. 49(23), 5283-5294 (2004).

25. J. Jiang et al., "Penetration kinetics of dimethyl sulphoxide and glycerol in dynamic optical clearing of porcine skin tissue in vitro studied by Fourier transform infrared spectroscopic imaging," J. Biomed. Opt. 13(2), 021105 (2008).

26. X. Xu and R. K. Wang, "Synergistic effect of hyperosmotic agents of dimethyl sulfoxide and glycerol on optical clearing of gastric tissue studied with near infrared spectroscopy," Phys. Med. Biol. 49(3), 457-468 (2004).

27. X. Xu and Q. Zhu, "Evaluation of skin optical clearing enhancement with azone as a penetration enhancer," Opt. Commun. 279(1), 223228 (2007).

28. D. Zhu et al., "Imaging dermal blood flow through the intact rat skin with an optical clearing method," J. Biomed. Opt. 15(2), 026008 (2010).

29. X. Wen et al., "Enhanced optical clearing of skin in vivo and optical coherence tomography in-depth imaging," J. Biomed. Opt. 17(6), 066022 (2012).

30. J. Wang et al., "Evaluation of optical clearing with the combined liquid paraffin and glycerol mixture," Biomed. Opt. Express 2(8), 2329-2338 (2011).

31. H. Shan et al., "Study on application of optical clearing technique in skin diseases," J. Biomed. Opt. 17(11), 115003 (2012).

32. K. Chen, Y. Liang, and Y. Zhang, "Study on reflection of human skin with liquid paraffin as the penetration enhancer by spectroscopy," J. Biomed. Opt. 18(10), 105001 (2013).

33. J. W. Wilson et al., "Optical clearing of archive-compatible paraffin embedded tissue for multiphoton microscopy," Biomed. Opt. Express 3(11), 2752-2760 (2012).

34. A. V. Rawlings and K. J. Lombard, "A review on the extensive skin benefits of mineral oil," Int. J. Cosmet. Sci. 34(6), 511-518 (2012).

35. J. Xi et al., "Diffractive catheter for ultrahigh-resolution spectraldomain volumetric OCT imaging," Opt. Lett. 39(7), 2016-2019 (2014).

36. W. Yuan et al., "Optimal operational conditions for supercontinuumbased ultrahigh-resolution endoscopic OCT imaging," Opt. Lett. 41(2), 250-253 (2016).

37. M. G. Ghosn et al., "Differential permeability rate and percent clearing of glucose in different regions in rabbit sclera," J. Biomed. Opt. 13(2), 021110 (2008).

38. M. G. Ghosn et al., "Permeability of hyperosmotic agent in normal and atherosclerotic vascular tissues," J. Biomed. Opt. 13(1), 010505 (2008).

39. M. G. Ghosn et al., "Permeation of human plasma lipoproteins in human carotid endarterectomy tissues: measurement by optical coherence tomography," J. Lipid Res. 52(7), 1429-1434 (2011). 
40. J. Xi, Y. Chen, and X. D. Li, "Characterizing optical properties of nano contrast agents by using cross-referencing OCT imaging," Biomed. Opt. Express 4(6), 842-851 (2013).

41. K. A. Vermeer et al., "Depth-resolved model-based reconstruction of attenuation coefficients in optical coherence tomography," Biomed. Opt. Express 5(1), 322-337 (2014).

42. N. Urganci, B. Akyildiz, and T. B. Polat, "A comparative study: the efficacy of liquid paraffin and lactulose in management of chronic functional constipation," Pediatr. Int. 47(1), 15-19 (2005).
43. N. Concin et al., "Mineral oil paraffins in human body fat and milk," Food Chem. Toxicol. 46(2), 544-552 (2008).

44. A. Patzelt et al., "In vivo investigations on the penetration of various oils and their influence on the skin barrier," Skin Res. Technol. 18(3), 364-369 (2012).

Biographies for the authors are not available. 